\title{
Sequential analysis of carbonaceous materials in Hayabusa-returned samples for the determination of their origin
}

\author{
Masayuki Uesugi ${ }^{1 *}$, Hiroshi Naraoka ${ }^{2}$, Motoo Ito $^{3}$, Hikaru Yabuta ${ }^{4}$, Fumio Kitajima², Yoshinori Takano ${ }^{5}$, Hajime Mita ${ }^{6}$, \\ Ichiro Ohnishi ${ }^{7}$, Yoko Kebukawa ${ }^{8}$, Toru Yada ${ }^{1}$, Yuzuru Karouji ${ }^{1}$, Yukihiro Ishibashi', Takaaki Okada ${ }^{1}$ and \\ Masanao Abe ${ }^{1}$
}

\begin{abstract}
Preliminary results of the analyses of five carbonaceous materials (particle size of approximately $50 \mu \mathrm{m}$ ) from the Hayabusa spacecraft sample catcher, including their texture, chemistry, and chemical/isotopic compositions, are summarized. The carbonaceous particles underwent sequential analysis using a series of microanalytical instruments located at several research institutes and universities. Collected particles were initially classified into four categories: two categories containing extraterrestrial silicate particles, one category containing metal and quartz particles consistent with contamination from the sample catcher or sample manipulation tools, and a final category containing carbonaceous particles. Analysis of this final category was the main focus of this study. Through examination of the carbonaceous materials, the appropriate analytical processes for sample transportation and handling were optimized to minimize sample damage and terrestrial contamination. Particles were investigated by transmission electron microscopy/scanning transmission electron microscopy, and Ca-carbonate inclusions were found in one particle. In a different particle, a heterogeneous distribution of silicon in a uniform C, N, and O matrix was found. Though further analysis is required for a strict determination of particle origin, the differences in the microstructure and elemental distribution of the carbonaceous particles suggest multiple origins.
\end{abstract}

Keywords: Hayabusa; Carbonaceous material; Sequential analysis; Microstructural observation by TEM/STEM

\section{Findings}

Introduction

More than 450 tiny particles have thus far been collected by the Hayabusa spacecraft sample catcher (Yada et al. 2014). These particles were initially classified into four categories based on their chemical composition obtained from analysis by a field emission scanning electron microscope (FE-SEM) with energy dispersion spectrometer (EDS). Particles classified as categories 1 and 2 were mainly composed of silicate materials and were confirmed as Itokawa regolith particles by the Hayabusa sample preliminary examination team (HASPET) (Ebihara et al. 2011; Nagao et al. 2011; Nakamura et al. 2011; Noguchi et al.

\footnotetext{
* Correspondence: uesugi@planeta.sci.isas.jaxa.jp

'Institute of Space and Astronautical Science (ISAS), Japan Aerospace Exploration Agency (JAXA), 3-1-1 Yoshinodai, Sagamihara, Kanagawa 252-5210, Japan

Full list of author information is available at the end of the article
}

2011; Tsuchiyama et al. 2011; Yurimoto et al. 2011). Category 4 particles were defined as particles containing synthetic material originating from the sample catcher, the micro-manipulator, and the clean chamber (e.g., aluminum flake, quartz glass, and stainless steel). However, the origins of category 3 particles, which were defined as particles composed mainly of carbon, were still unknown. The first analysis of category 3 particles (hereafter, first examination) was performed in parallel with the preliminary examination of silicate materials (categories 1 and 2). The difficulties in handling and/or processing of these small carbonaceous samples without introducing terrestrial contamination resulted in the collection of insufficient information from the first examination to explore the origin of category 3 particles. Kitajima et al. (2011) and Naraoka et al. (2012) investigated the existence of organic materials on the surface of category 1 and 2 particles during preliminary 
examinations of these silicate materials. They concluded that organic material, such as that found in the category 3 particles, was not detected on the surface of category 1 and 2 particles. Thus, there is currently no information on the origin of category 3 particles.

After preliminary examination of the silicate material, new analytical facilities and members were introduced to the HASPET for reexamination of the category 3 particles (hereafter, second examination). In order to examine the origin of category 3 particles by utilizing different chemical and physical information, the handling processes of the carbonaceous particles were improved and multiple microanalyses were applied to single particles. In this paper, the newly developed analytical scheme for analysis of the category 3 particles, including sample processing and analytical techniques developed during the first and second examinations, are reported. In addition, the results of the second examination of the category 3 particles are briefly summarized.

\section{Description of category 3 particles}

Fifty-eight out of 459 particles were classified as category 3 by FE-SEM with EDS analysis at the Extraterrestrial Sample Curation Center of JAXA (ESCuC/JAXA). Six particles were lost during sample transfer from the SEM holder to the storage slide glass using an electrostatically controlled manipulation system (for a complete description, see Yada et al. 2014). Thus, 52 category 3 particles were stored in the clean chamber at ESCuC/JAXA under purified nitrogen $\left(\mathrm{N}_{2}\right)$ gas. Category 3 samples were found to be mainly composed of carbon $(\mathrm{C})$, nitrogen $(\mathrm{N})$, and oxygen $(\mathrm{O})$, with trace amounts of fluorine $(\mathrm{F})$ and sulfur (S). The sizes of the category 3 particles were similar to that of category 1 and 2 particles, ranging from 15 to $208 \mu \mathrm{m}$ in diameter.

Figure 1 shows backscattered electron (BSE) images of selected category 3 particles. From the BSE images, the category 3 particles were further divided into three subcategories, types 1 to 3 , based on their texture and chemical composition. Type 1 was defined as blocky particles, which were found to have irregular shapes and small white grains on their surfaces; the grains were found to be composed of silicate, iron sulfide, stainless steel, and aluminum (Al). These blocky type 1 particles were the largest subcategory within category 3 (38 out of 52 particles). The silicate grains were found to be olivine, pyroxene, plagioclase, iron sulfide, and potassium feldspar. These minerals were also components of category 1 and 2 particles. Therefore, it was assumed that these small silicate grains were also probably fragments of Itokawa particles. The small surface grains of stainless steel and $\mathrm{Al}$ were most likely contaminants from the sample catcher or the clean chamber. Type 1 particles also often included chloride $(\mathrm{Cl})$ salts such as sodium chloride $(\mathrm{NaCl})$, potassium chloride $(\mathrm{KCl})$, and calcium chloride $\left(\mathrm{CaCl}_{2}\right)$.

Type 2 particles were defined by their fibrous shape, and 6 out of 52 category 3 particles were defined as type 2 . Type 3 particles were deemed faint type, having a very smooth surface marked by very few tiny surface particles. All type 3 particles had an $\mathrm{N}$ peak in their EDS spectrum, and 8 out of 52 particles were classified as this particle type.

The differences in the texture and chemical composition of each defined particle type within category 3 might indicate a different origin for each particle type. Alternatively, these differences may indicate that these particles were initially from the same source but underwent different irradiation processing or other alterations before their recovery from the sample catcher. Table 1 shows the characteristics of all category 3 particles. Pictures and other information on category 3 particles are available in the sample catalogue of Hayabusa-returned samples (http://hayabusaao.isas.jaxa.jp/catalog/cat3/).

\section{Sample preparation and analysis method}

For the preliminary examinations of category 1 and 2 particles (i.e., silicate materials), the samples were fixed on the top of a 5- $\mu \mathrm{m}$ fiber with epoxy resin or glycol phthalate (e.g., Nakamura et al. 2011) to facilitate sample handling and transfer. However, these adhesive materials, which included water and ethanol, could be a source of potential contamination and/or damage during the handling of category 3 (carbonaceous) particles. As such, category 3 samples should be handled in a completely dry environment, without adhesive, in order to avoid sample damage and contamination. Thus, for the first examination, category 3 particles were transferred using a diamond or quartz sample-holder under a purified $\mathrm{N}_{2}$ atmosphere. However, the samples were difficult to handle using the manipulator without any adhesive material or liquid, resulting in the loss of a particle (RA-QD02-0008) during handling.

For the second examination, samples were fixed on 0.3-mm-thick metal plates (gold ( $\mathrm{Au}$ ) or indium (In)) on the SEM sample holder (Figure 2). The samples were pressed onto the $\mathrm{Au}$ or In plates by 5 -mm-thick sapphire windows installed in a special pressure apparatus so that samples could be observed through the glass during pressing. Au or In were chosen as sample substrates to minimize potential contamination and charging artifacts during SEM, NanoSIMS (secondary ion mass spectroscopy), and time-of-flight (ToF)-SIMS analyses.

The subset of category 3 samples allocated for the first and second preliminary examinations were RA-QD020008, RA-QD02-0120, RA-QD02-0180, RB-QD04-0001, RB-QD04-0037-01, and RB-QD04-0047-02 (Figure 1). 


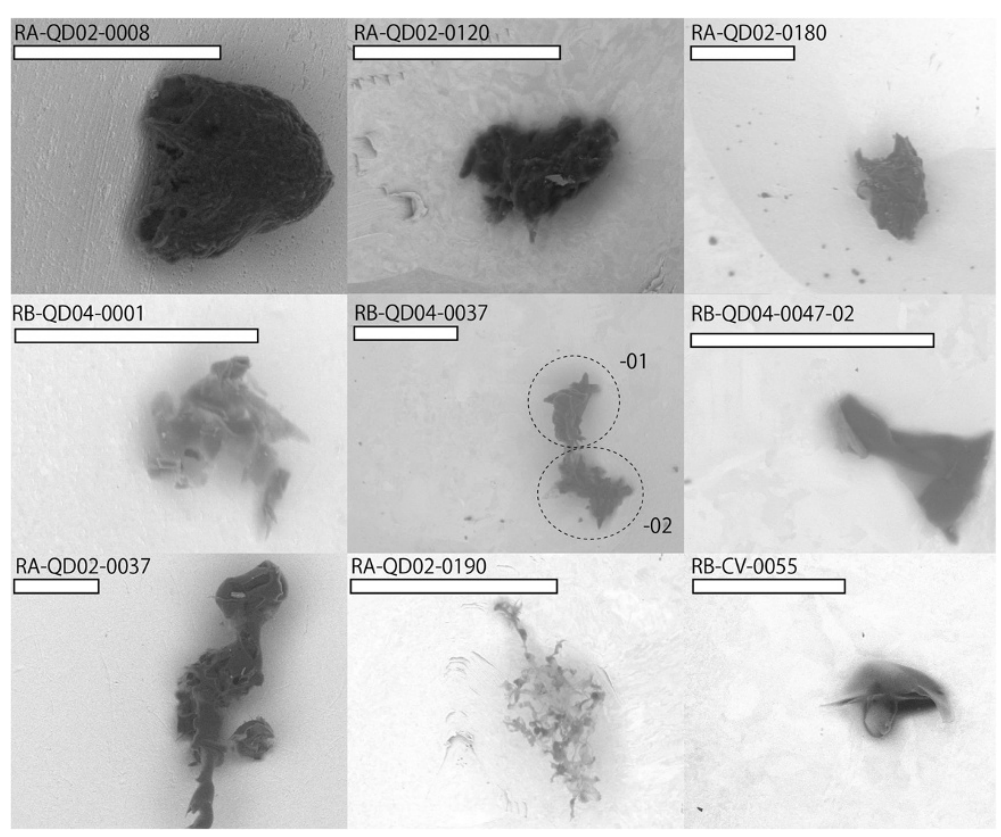

Figure 1 Backscattered electron images of category 3 particles. White bars show $50 \mu \mathrm{m}$. Particles allocated to the first and second examinations are shown in the top and middle rows of the figure. RA-QD02-0190 is classified as fibrous type (type 2), and RB-QD04-0001 and RB-CV-0055 are classified as faint type (type 3).

All samples were first analyzed by FE-SEM-EDS (Hitachi S-4300E/N, SU6600, ESCuC/JAXA, Hitachi, Tokyo, Japan) during the characterization process required for nominal curation, referred to as initial description' (Yada et al. 2014). This initial description separated the returned samples into the four categories described above.

Four particles, RA-QD02-0008, RA-QD02-0120, RBQD04-0001, and RB-QD04-0047-02, were previously chosen to be analyzed in the first examination and, thus, had been exposed to the terrestrial atmosphere. Two of these particles, RA-QD02-0008 and RB-QD040001, were analyzed by Fourier transform infrared spectroscopy (FT-IR) and Raman spectroscopy in the first examination (Kitajima et al. 2014), while RBQD04-0047-02 and RA-QD02-0120 were returned to ESCuC/JAXA without any analyses from the first examination. Thus, these samples were minimally affected by brief atmospheric exposure during the first examination. Samples allocated to the first examination were stored for 6 months in a purified $\mathrm{N}_{2}$ desiccator after analysis and then allocated to the second examination.

Except for RA-QD02-0008, particles allocated to the first examination were pressed on an $\mathrm{Au}$ plate under terrestrial atmosphere at the beginning of the second examination. The two new particles allocated solely to the second examination, RA-QD02-0180 and RBQD04-0037-01, were pressed onto In plates under purified $\mathrm{N}_{2}$ gas. Several types of microanalysis instruments located in different laboratories (NanoSIMS ion microprobe: CAMECA NanoSIMS $50 \mathrm{~L}$ at Kochi Institute for Core Sample Research/JAMSTEC, ToF-SIMS: ULVAC-PHI TRIFT III at Nagoya University, FT-IR: JASCO IRT-5000 at ESCuC/JAXA and Perkin-Elmer Spectrum One at Kyushu University, Raman spectroscopy: JASCO NRS-5100 at ESCuC/JAXA and JEOL JRS System 2000 at Kyushu University) were used on the pressed samples. If evidence of extraterrestrial material (i.e., isotopic anomalies of $\mathrm{H}, \mathrm{C}$, and $\mathrm{N}$ in organics) was found, the second examination was immediately closed and a more detailed analysis commenced.

After the first analyses of the pressed samples, ultrathin sections (UTSs) of RA-QD02-0120 and RB-QD040047-02 $(20 \mu \mathrm{m} \times 1 \mu \mathrm{m} \times 100 \mathrm{~nm})$ were extracted by a focused ion beam (FIB, Hitachi FB2200, ESCuC/JAXA). These UTSs were mounted on copper $(\mathrm{Cu})$ grids by tungsten deposition, and the $\mathrm{Cu}$ grids were fixed on polyurethane elastomers during sample transfer. X-rayabsorption near edge structure (XANES) (ALS/Berkley and UVSOR/IMS) and (scanning) transmission electron microscopy ((S)TEM, JEOL JEM-2800, JEM-2100 F, JEOL Ltd., Tokyo, Japan) were examined to further analyze the UTSs. Characteristics of the samples and a summary of the applied analyses are reported in Table 2. Details of NanoSIMS (Ito et al. 2014), ToF-SIMS (Naraoka et al. 2014), FT-IR and Raman spectroscopy (Kitajima et al. 
Table 1 List of category 3 particles

\begin{tabular}{|c|c|c|c|c|}
\hline Name & $\operatorname{Size}^{\mathrm{a}}(\mu \mathrm{m})$ & Type & Elements $^{\mathrm{b}}$ & Surface grains \\
\hline RA-QD02-0008 & 50 & Type1: blocky & $\mathrm{C}, \mathrm{N}, \mathrm{O}$ & $\mathrm{Al}$ \\
\hline RA-QD02-0012 & 100 & Type1: blocky & $\mathrm{C}, \mathrm{O}$ & FeS \\
\hline RA-QD02-0018 & 50 & Type1: blocky & $\mathrm{C}, \mathrm{O}, \mathrm{Cl}$ & Al, ol, Ipx \\
\hline RA-QD02-0037 & 60 & Type1: blocky & $\mathrm{C}, \mathrm{O}$ & ol, lpx \\
\hline RA-QD02-0040-01 & 30 & Type1: blocky & $\mathrm{C}, \mathrm{O}, \mathrm{Si}$ & Lpx \\
\hline RA-QD02-0078 & 100 & Type1: blocky & $\mathrm{C}, \mathrm{O}, \mathrm{Cl}, \mathrm{F}, \mathrm{Si}$ & Lpx \\
\hline RA-QD02-0091 & 30 & Type1: blocky & $\mathrm{C}, \mathrm{O}, \mathrm{Si}, \mathrm{Cl}$ & \\
\hline RA-QD02-0120 & 26 & Type1: blocky & $\mathrm{C}, \mathrm{O}$ & SUS \\
\hline RA-QD02-0134 & 21 & Type1: blocky & $\mathrm{K}, \mathrm{Cl}$ & \\
\hline RA-QD02-0180 & 55 & Type1: blocky & $\mathrm{C}, \mathrm{O}, \mathrm{K}, \mathrm{Na}, \mathrm{Cl}$ & \\
\hline RA-QD02-0181 & 55 & Type1: blocky & $\mathrm{C}, \mathrm{N}, \mathrm{O}, \mathrm{Na}, \mathrm{K}, \mathrm{Cl}$ & \\
\hline RA-QD02-0182 & 46 & Type1: blocky & $\mathrm{C}, \mathrm{N}, \mathrm{O}, \mathrm{Na}, \mathrm{K}, \mathrm{Cl}$ & \\
\hline RA-QD02-0183 & 38 & Type1: blocky & $\mathrm{C}, \mathrm{N}, \mathrm{O}, \mathrm{Na}, \mathrm{K}, \mathrm{Cl}$ & \\
\hline RA-QD02-0190 & 58 & Type2: fibrous & $C, N$ & \\
\hline RA-QD02-0222 & 121 & Type2: fibrous & $\mathrm{C}, \mathrm{O}, \mathrm{Mg} \mathrm{Si}$ & \\
\hline RB-CV-0005 & 37 & Type1: blocky & $\mathrm{C}, \mathrm{Na}, \mathrm{Cl}$ & \\
\hline RB-CV-0006 & 27.9 & Type1: blocky & $\mathrm{C}, \mathrm{N}, \mathrm{O}$ & \\
\hline RB-CV-0007 & 43.1 & Type1: blocky & $\mathrm{C}, \mathrm{N}, \mathrm{O}$ & \\
\hline RB-CV-0008 & 56.1 & Type1: blocky & $\mathrm{C}, \mathrm{N}, \mathrm{O}$ & \\
\hline RB-CV-0012 & 73.2 & Type2: fibrous & $\mathrm{C}, \mathrm{N}, \mathrm{O}$ & \\
\hline RB-CV-0017 & 51.7 & Type1: blocky & $\mathrm{C}, \mathrm{N}, \mathrm{O}$ & \\
\hline RB-CV-0019 & 32.6 & Type3: faint & $\mathrm{C}, \mathrm{N}, \mathrm{O}$ & \\
\hline RB-CV-0020 & 68.6 & Type1: blocky & $\mathrm{C}, \mathrm{O}$ & \\
\hline RB-CV-0021 & 49.3 & Type2: fibrous & $\mathrm{C}, \mathrm{O}$ & \\
\hline RB-CV-0027 & 59 & Type1: blocky & $\mathrm{C}, \mathrm{N}, \mathrm{O}$ & \\
\hline RB-CV-0029 & 86 & Type1: blocky & $\mathrm{C}, \mathrm{O}, \mathrm{Na}, \mathrm{Cl}$ & $\mathrm{Pl}$ \\
\hline RB-CV-0031 & 61 & Type2: fibrous & $\mathrm{C}, \mathrm{N}, \mathrm{O}$ & \\
\hline RB-CV-0032 & 59 & Type3: faint & $\mathrm{C}, \mathrm{N}, \mathrm{O}$ & \\
\hline RB-CV-0035 & 25 & Type1: blocky & $\mathrm{C}, \mathrm{N}, \mathrm{O}, \mathrm{Na}, \mathrm{Cl}$ & hpx, Al \\
\hline RB-CV-0041 & 49 & Type1: blocky & $\mathrm{C}, \mathrm{N}, \mathrm{O}, \mathrm{Na}, \mathrm{Cl}$ & \\
\hline RB-CV-0047 & 48 & Type1: blocky & $\mathrm{C}, \mathrm{O}, \mathrm{Al}, \mathrm{Ca}, \mathrm{Si}$ & \\
\hline RB-CV-0049 & 37 & Type3: faint & $\mathrm{C}, \mathrm{N}, \mathrm{O}$ & \\
\hline RB-CV-0052 & 40 & Type1: blocky & $\mathrm{C}, \mathrm{O}, \mathrm{Na}, \mathrm{Cl}$ & \\
\hline RB-CV-0055 & 45 & Type3: faint & $\mathrm{C}, \mathrm{N}, \mathrm{O}, \mathrm{Al}$ & \\
\hline RB-CV-0065 & 33 & Type1: blocky & $\mathrm{C}, \mathrm{N}, \mathrm{O}$ & \\
\hline RB-CV-0066 & 29 & Type1: blocky & $\mathrm{C}, \mathrm{N}, \mathrm{O}, \mathrm{Na}, \mathrm{Cl}$ & \\
\hline RB-CV-0068 & 55 & Type3: faint & $\mathrm{C}, \mathrm{N}, \mathrm{O}$ & $\mathrm{Ol}$ \\
\hline RB-CV-0074 & 26 & Type1: blocky & $\mathrm{C}, \mathrm{N}, \mathrm{O}, \mathrm{Na}, \mathrm{Cl}$ & $\mathrm{Al}$ \\
\hline RB-CV-0077 & 100 & Type3: faint & $\mathrm{C}, \mathrm{N}, \mathrm{O}$ & \\
\hline RB-CV-0078 & 208 & Type2: fibrous & $\mathrm{C}, \mathrm{O}$ & \\
\hline RB-CV-0079 & 68 & Type1: blocky & $\mathrm{C}, \mathrm{N}, \mathrm{O}$ & K-feld \\
\hline RB-CV-0080 & 83 & Type1: blocky & $\mathrm{C}, \mathrm{N}, \mathrm{O}$ & K-feld \\
\hline RB-QD04-0001 & 19 & Type3: faint & $\mathrm{C}, \mathrm{N}, \mathrm{O}$ & $\mathrm{Ol}, \mathrm{Al}$ \\
\hline RB-QD04-0031 & 64 & Type1: blocky & $\mathrm{C}, \mathrm{O}, \mathrm{Al}$ & \\
\hline
\end{tabular}


Table 1 List of category 3 particles (Continued)

\begin{tabular}{llcc}
\hline RB-QD04-0035 & 59 & Type1: blocky & C, N, O, Na, K, Cl, Al \\
RB-QD04-0037-01 & 48 & Type1: blocky & C, N, O \\
RB-QD04-0037-03 & 15 & Type3: faint & C, N, O \\
RB-QD04-0047-01 & 66 & Type1: blocky & C, O \\
RB-QD04-0047-02 & 28 & Type1: blocky & C, O \\
RB-QD04-0048 & 56 & Type1: blocky & C, O \\
RB-QD04-0052 & 96 & Type1: blocky & C, F, O, Al, Ti \\
RB-QD04-0078 & 19 & Type1: blocky & C, O \\
\hline
\end{tabular}

${ }^{a}$ Length of the major axis; ${ }^{b}$ found in FE-SEM-EDS analysis. ol, olivine; pl, plagioclase; K-fld, K feldspar; hpx, high-Ca pyroxene; Ipx, low-Ca pyroxene; SUS, stainless steel; FeS, iron sulfide.

2014), and XANES (Yabuta et al. 2014) analyses are described in papers in this issue.

Both pressed samples and UTSs were transferred by parcel delivery service to each institute or university within a $\mathrm{N}_{2}$-purged environment. Prior to the analysis of category 3 particles, analysis procedures, including sample transfer, were tested using carbonaceous particles collected at the spacecraft and fairing assembly building (SFA) of the Tanegashima Space Center/JAXA (hereafter SFA particle), insoluble organic matter (IOM) of the Antarctic meteorite A-881458 (CM2), and synthetic $\mathrm{Mg}-\mathrm{Fe}-\mathrm{Ni}$ olivine particles. All test particles were in the same size range as the Hayabusareturned samples. NanoSIMS isotope analysis of $\mathrm{H}, \mathrm{C}$, and $\mathrm{N}$ measured terrestrial isotopic ratios for the SFA particles and extraterrestrial isotopic signatures for the IOM from A-881458 (Ito et al. 2014). From this data, it was confirmed that the sample handling system and transportation process were suitable for these organic samples.

\section{Evaluation of possible sample damage and contamination during analysis}

Evaluation of sample damage by SIMS analysis The purpose of this study was to apply several microanalytical techniques to a single particle to suppress any errors caused by the instruments used during the sequential analysis. In order to investigate the change of a sample's isotopic ratio as a function of sample analysis sequence order, RBQD04-0047-02 was analyzed by NanoSIMS before and after ToF-SIMS analysis. The isotopic values of the samples from NanoSIMS analysis obtained prior to ToF-SIMS analysis were $\delta \mathrm{D}=+103 \%$ (vs SMOW), $\delta^{13} \mathrm{C}=+3 \%$ (vs PDB), and $\delta^{15} \mathrm{~N}=-4 \%$ (vs air), and the values obtained after ToF-SIMS analysis were $\delta \mathrm{D}=+177 \%$, $\delta^{13} \mathrm{C}=+14 \%$, and $\delta^{15} \mathrm{~N}=+6 \%$ (Ito et al. 2014). The isotopic ratios tended to be slightly enriched in the heavier isotope after ToF-SIMS analysis. However, the change in $\delta \mathrm{D}$ was smaller than the expected isotopic anomalies found in the organic material of ordinary chondrites $(\delta \mathrm{D}$ of approximately $+4000 \%$, e.g., Alexander et al.

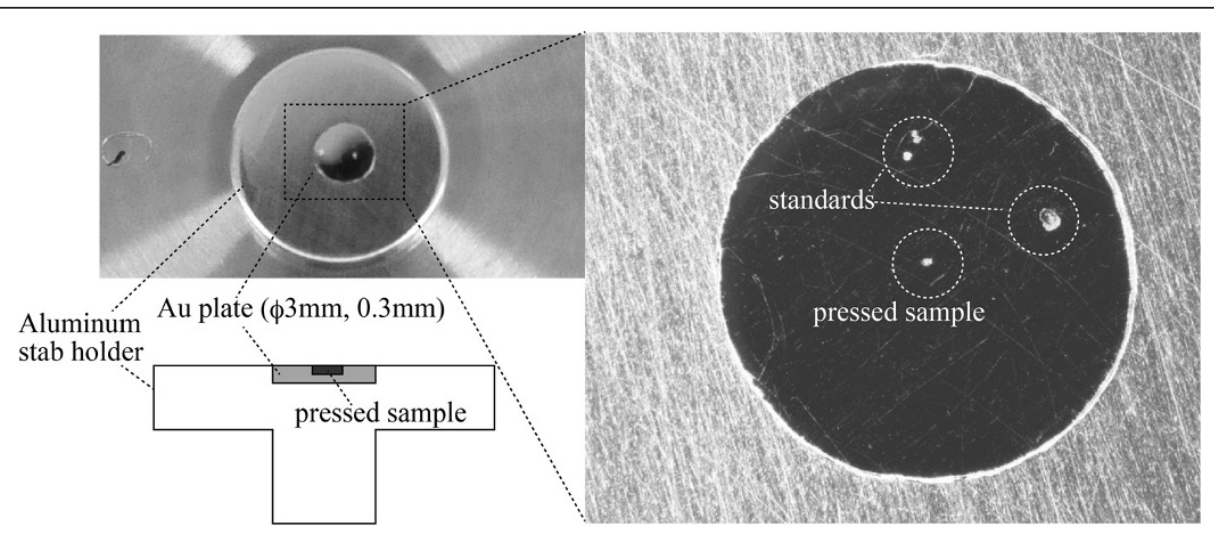

Figure 2 A schematic illustration and pictures of the sample holder. Upper left: a picture of the top view of the sample holder. An Au disk, ب3-mm and 0.3-mm thick, is fixed onto an Al-stub holder. Lower left: a schematic illustration of the side view of the sample holder. Right: an enlarged view of the Au disk. The sample was pressed and fixed at the center of the disk. Standards for isotopic analysis by NanoSIMS were also fixed near the edge of the Au disk and are marked in the figure. 
Table 2 Details of the samples and analysis

\begin{tabular}{|c|c|c|c|c|c|}
\hline Sample name & $\operatorname{Size}^{\mathrm{a}}(\mu \mathrm{m})$ & Pressed on & Type & Elements $^{\mathbf{b}}$ & Analysis flow \\
\hline RA-QD02-0008 & 50 & (Lost) & Blocky & $\mathrm{C}, \mathrm{N}, \mathrm{O}, \mathrm{Al}$ & FT-IR, Raman (Lost) \\
\hline RA-QD02-0120 & 26 & $\mathrm{Au}$ & Blocky & $\mathrm{C}, \mathrm{O}$ & NanoSIMS, FT-IR, Raman, XANES, TEM \\
\hline RA-QD02-0180 & 55 & $\ln$ & Blocky & $\mathrm{C}, \mathrm{O}, \mathrm{K}, \mathrm{Na}, \mathrm{Cl}$ & ToF-SIMS, NanoSIMS, Raman \\
\hline RB-QD04-0001 & 19 & $\mathrm{Au}$ & Faint & $\mathrm{C}, \mathrm{N}, \mathrm{O}$ & NanoSIMS, FT-IR, Raman \\
\hline RB-QD04-0037-01 & 48 & $\ln$ & Blocky & $\mathrm{C}, \mathrm{N}, \mathrm{O}$ & FT-IR, ToF-SIMS \\
\hline RB-QD04-0047-02 & 28 & $\mathrm{Au}$ & Blocky & $\mathrm{C}, \mathrm{O}$ & NanoSIMS, FT-IR, Raman, ToF-SIMS, NanoSIMS, XANES, TEM \\
\hline S01 (SFA particle) & 30 & $\mathrm{Au}$ & Blocky & $\mathrm{C}, \mathrm{O}$ & NanoSIMS \\
\hline IOM1 (A-881458, CM2) & 50 & $\mathrm{Au}$ & Blocky & & NanoSIMS, FT-IR, Raman, XANES, TEM \\
\hline NI1 (synthetic Mg-Fe-Ni olivine) & 50 & $\mathrm{Au}$ & - & - & XANES, TEM \\
\hline
\end{tabular}

aLength of the major axis; ${ }^{\text {b }}$ found in FE-SEM-EDS analysis.

2007). Therefore, measurement of a hydrogen isotopic anomaly should be possible after ToF-SIMS analysis. Contrastingly, the results of ToF-SIMS analysis were seriously altered by interference from Cesium (Cs), the primary ion beam of NanoSIMS, and by $\mathrm{Au}$ deposits from sputtering during the NanoSIMS analysis.

The effect of the ToF-SIMS analysis on FT-IR analysis was also investigated. Figure 3 a shows the difference in the FT-IR spectrum of RB-QD04-0037-01 before and after ToF-SIMS analysis. The measured spectrum was greatly altered by ToF-SIMS analysis: a major peak between 2,500 and $2,000 \mathrm{~cm}^{-1}$ disappeared, and the overall spectrum was significantly smoothed.

Evaluation of contamination through the FIB UTS extraction process Contamination transferred by the FIB from previous sample residue was evaluated by pressing a $50-\mu \mathrm{m}$ fragment of the IOM of A-881458 (CM2) onto an Au plate and extracting a UTS from this pressed sample with the FIB. Before and after fabricating a UTS from the IOM, UTSs of synthetic Mg-Fe-Ni olivine were also extracted. The synthetic $\mathrm{Mg}-\mathrm{Fe}-\mathrm{Ni}$ olivine UTSs were analyzed by O-XANES. No changes were observed in the synthetic $\mathrm{Mg}-\mathrm{Fe}-\mathrm{Ni}$ olivine peak signals measured by O-XANES before and after the IOM fabrication (Figure $3 \mathrm{~b}$ ). Thus, the transfer of the carbonaceous material via the FIB was likely negligible and should not affect subsequent analyses of the carbonaceous material.

Evaluation of sample damage by electron beam of TEM/STEM on XANES spectra Yabuta et al. (2014) reported a change in the C-XANES spectra of RA-QD020120 before and after TEM analysis attributed to sample damage by the electron beam. This effect was reported in a previous study (e.g. De Gregorio et al. 2010) and should be considered in the sequential analysis.
Reconstruction of analytical flow for carbonaceous materials Through the discussed analyses, the flow of the sequential analysis was constructed using FT-IR, ToFSIMS, NanoSIMS, Raman spectroscopy, XANES and TEM/STEM to minimize sample contamination during analysis (Table 3 ). The reconstructed flow was applied to the analysis of the previously unanalyzed RB-QD040037-01 and RA-QD02-0180 samples allocated to the second examination.

\section{Analyses applied to each particle in the first and second examinations}

Sample names, categories, sizes, observed EDS peaks, and techniques used for this study are summarized in Table 2. RA-QD02-0008 was analyzed only in the first examination. FE-SEM-EDS analysis identified C, N, O, and $\mathrm{Al}$ peaks in the sample. After the FT-IR and Raman spectroscopy measurements, the sample was lost during preparation for ToF-SIMS analysis.

RA-QD02-0120 was analyzed by NanoSIMS, FT-IR, Raman spectroscopy, XANES, and TEM in the second examination. This particle was analyzed using FT-IR and Raman spectroscopy after NanoSIMS analysis; thus, the spectra were probably altered (smoothed) by the effect described previously.

RA-QD02-0180 was only analyzed during the second examination and was found to have $\mathrm{NaCl}$ and $\mathrm{KCl}$ peaks as well as $\mathrm{C}$ and $\mathrm{O}$ peaks in the FE-SEM-EDS spectrum. The particle was carefully pressed onto an In plate in a purified $\mathrm{N}_{2}$ environment and analyzed by ToF-SIMS. In order to avoid damage to the chlorides in the sample from atmospheric exposure, FT-IR analysis was not conducted on this sample. Currently, atmospheric exposure is unavoidable during FT-IR analysis at ESCuC/JAXA, though improvements to this system are being planned to address this issue.

RB-QD04-0001 was analyzed during the first examination by FT-IR and Raman spectroscopy. In the second examination, the particle was analyzed by NanoSIMS, FT- 

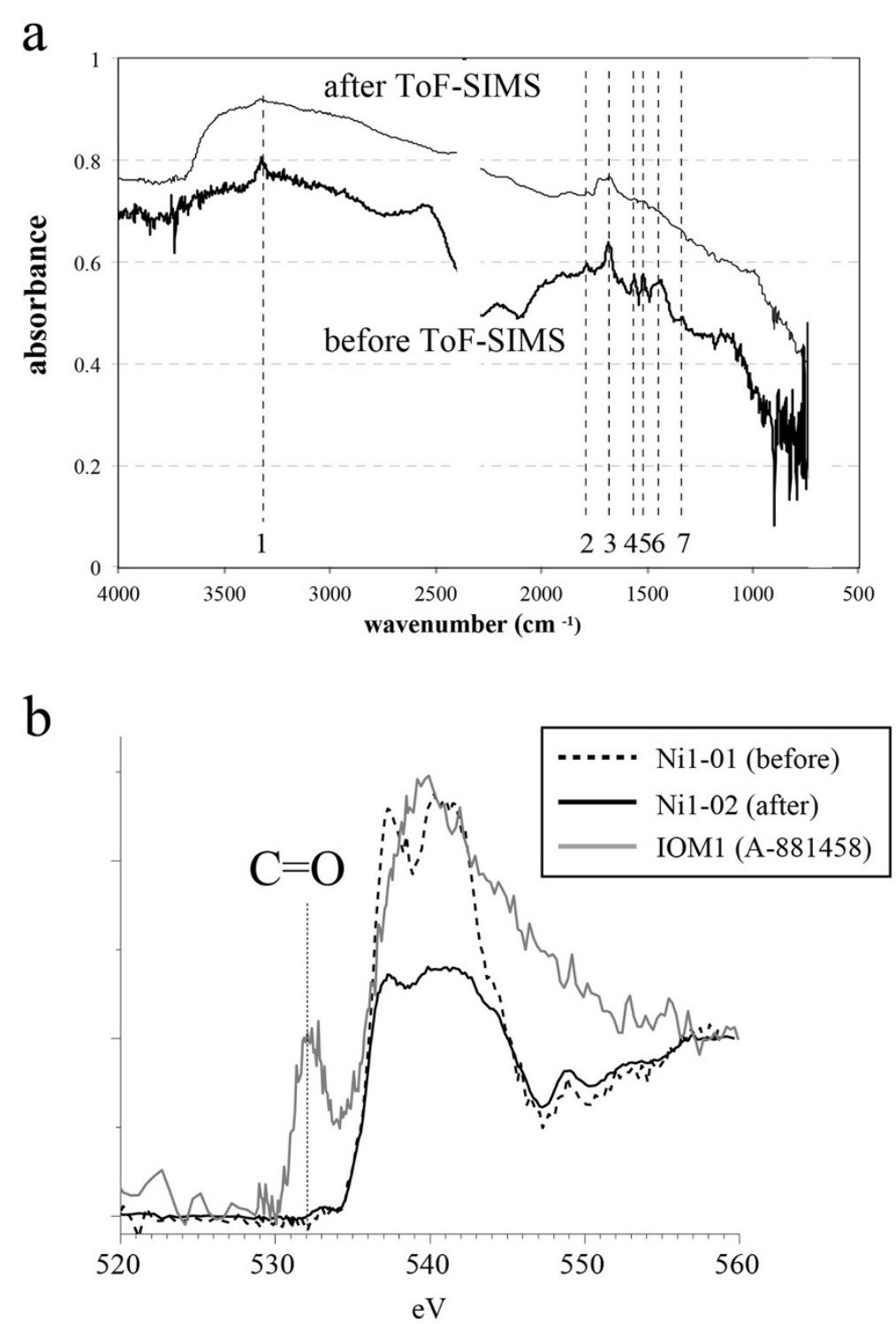

Figure 3 Results of the tests for sample damage by ToF-SIMS and for FIB cross-contamination. (a) Change of the FT-IR spectrum before and after ToF-SIMS analysis. Dashed lines show where the peak positions appear in the spectrum before ToF-SIMS analysis: (1) $3,300 \mathrm{~cm}^{-1}-\mathrm{N}-\mathrm{H}$ stretching; (2) 1,800 and (3) $1,680 \mathrm{~cm}^{-1}-\mathrm{C}=\mathrm{O}$ stretching; (4) 1,560 and (5) $1,510 \mathrm{~cm}^{-1}-\mathrm{N}-\mathrm{H}$ deformation; (6) $1,450 \mathrm{~cm}^{-1}-\mathrm{N}-\mathrm{H}$ bending; (7) $1,330 \mathrm{~cm}^{-1}$ C-N stretching. (b) O-XANES spectrum of UTSS of a synthetic Mg-Fe-Ni olivine sample before and after FIB fabrication of the IOM (A-881458, CM2) UTS. Vertical axis shows absorbance normalized by direct beam. A dashed line at $532 \mathrm{eV}$ shows C-O bond absorption from IOM. Ni01-01 and Ni01-02 are the spectra from the UTSs before and after the FIB fabrication of IOM.

IR, and Raman spectroscopy. This was the only the particle classified into the category 3 type 3 (faint type) particles among the particles previously analyzed. During the NanoSIMS analysis, the pressed sample on the $\mathrm{Au}$ plate was almost completely sputtered away due to its small size. Thus, the sample could not be analyzed further.

RB-QD04-0037-01 was also only analyzed during the second examination. FE-SEM-EDS analysis showed C, $\mathrm{N}$, and $\mathrm{O}$, and olivine inclusions on the surface of the particle. The sample was pressed onto an In plate and analyzed by FT-IR and ToF-SIMS.

The FE-SEM-EDS spectrum of RB-QD04-0047-02 was found to contain only $\mathrm{C}$ and $\mathrm{O}$ peaks. This particle was also analyzed by NanoSIMS, FT-IR, Raman spectroscopy, ToF-SIMS, XANES, and TEM/STEM. This particle was very hard compared to other particles, as its shape did not change during handling or after pressing onto the $\mathrm{Au}$ metal substrate. In comparison, other particles were deformed or crushed during this pressing process. 
Table 3 Reconstructed analytical flow and effects on other measurements

\begin{tabular}{llll}
\hline Order & Method & Data & Sample damage \\
\hline 1 & FT-IR & Molecular structure & - \\
2 & ToF-SIMS & $\begin{array}{l}\text { Elemental mapping and analysis of } \\
\text { molecular species }\end{array}$ & $\begin{array}{l}\text { Smoothing of FT-IR spectra, moderate change of isotope ratio of NanoSIMS } \\
\text { analysis (Ito et al. 2014) }\end{array}$ \\
3 & NanoSIMS & Isotope ratio and mapping & Large disturbance of ToF-SIMS result by CS + implantation (Naraoka et al. 2014) \\
5 & Raman spectroscopy & Molecular structure & Thermal damage by laser (not evaluated in this paper) \\
6 & XANES & Molecular structure & Sample destruction by FIB sectioning \\
& TEM/STEM & Nanoscale texture and elemental & Sample destruction by FIB sectioning, electron beam damage on molecular \\
\end{tabular}

\section{Results of TEM/STEM analysis}

Figure 4 shows the TEM/STEM images of RA-QD020120 and RB-QD04-0047-02. Examination of the UTS of RA-QD02-0120 revealed Ca-carbonate inclusions up to $300 \mathrm{~nm}$ in diameter. The matrix of the carbonaceous material showed uniform $\mathrm{C}, \mathrm{N}$, and $\mathrm{O}$ distributions. In the UTS of RB-QD04-0047-02, no distinct inclusions were apparent. In addition, only a heterogeneous distribution of $\mathrm{Si}$ was observed in the carbonaceous material from the EDS map.

\section{Discussion and future work}

Sequential analysis of category 3 particles allowed us to measure a number of chemical and structural characteristics, which could be related to the origin of these particles. No isotopic anomalies were found, suggesting these particles were not of extraterrestrial origin (Ito et al. 2014). However, as this finding does not immediately discount extraterrestrial origin, further analysis of this category for terrestrial contaminants was deemed necessary. FT-IR and Raman spectroscopy spectra of all samples showed differences between these category 3 samples and the meteoritic IOM (Kitajima et al. 2014), also suggesting that the category 3 particles are of terrestrial origin.

The UTS of the IOM of A-881458 was investigated using TEM/STEM (Figure 5). A nano-globule was found in the pressed IOM similar to previous findings from extraterrestrial matter (e.g., Nakamura-Messenger et al. 2002; Garvie and Buseck 2004; Nakamura-Messenger et al. 2006; Matsumoto et al. 2013). Thus, particles of extraterrestrial origin may be identified by the presence of these globules in the UTSs. These globules were not detected in any of the UTSs of category 3 particles. Together, the lack of an extraterrestrial isotopic signature, the difference between the FT-IR and Raman spectroscopy spectra of category 3 particles and meteoritic IOM, and the lack of nano-globules indicate a low possibility that category 3 particles are of extraterrestrial origin.

A Si distribution was detected in RB-QD04-0047-02 by TEM observation indicating that these particles are probably not of biological origin. ToF-SIMS analysis of RB-QD04-0037-01 also showed Si distribution over a wide area on the surface of the pressed sample (Naraoka et al. 2014). Thus, silicon rubber was considered as a possible origin of the sample. However, FE-SEM-EDS showed the presence of N in RB-D04-0037-01, which was not apparent in the FE-SEM-EDS spectrum of a silicon-rubber standard sample. A uniform distribution of $\mathrm{N}, \mathrm{C}$, and $\mathrm{O}$ was also found utilizing a STEM-EDS elemental map of RA-QD02-0120 and RB-QD04-0047-02. The nitrogen signature in these samples was also detected by XANES analyses (Yabuta et al. 2014). These results suggest that the origin of these particles may not be silicon rubber or that the properties of these particles were altered from their original material by heat or cosmic ray irradiation during space flight.

Ca-carbonate inclusions were detected on the UTS of RA-QD02-0120 by TEM/STEM. However, Si was not found in the STEM-EDS map of the particle, indicating that this particle is different in origin from both RBQD04-0037-01 and RB-QD04-0047-02. Though their exact origins are still uncertain, this data suggests that there may be several different category 3 particle sources. Due to its small mass, there is insufficient information on RB-QD04-0001 for the determination of its source.

In order to determine the origin of category 3 particles, possible contaminants, such as fluoro-rubber, silicon rubber, and Vectran, as well as extraterrestrial organic matter, must be characterized using the same analytical techniques used to analyze the Hayabusa-returned samples. Vectran, a polyarylate fiber, was used in the outer cover of the Hayabusa spacecraft sampler horn. Fluoro-rubber is used for the gloves of the clean chambers where the Hayabusa-returned samples are stored. Silicon rubber is generally used for the seal material of clean rooms. Particles collected from contamination coupons, which were exposed in the clean room during examinations of the Hayabusa 2 spacecraft (unpublished data), will also be analyzed. Examination of the contamination coupons will allow examination of potential contaminants prior to launch of the Hayabusa 2 spacecraft. By comparing the analytical results of potential contaminants and category 3 particles, the precise origin of category 3 particles may be determined. The effect of the long-term exposure of 

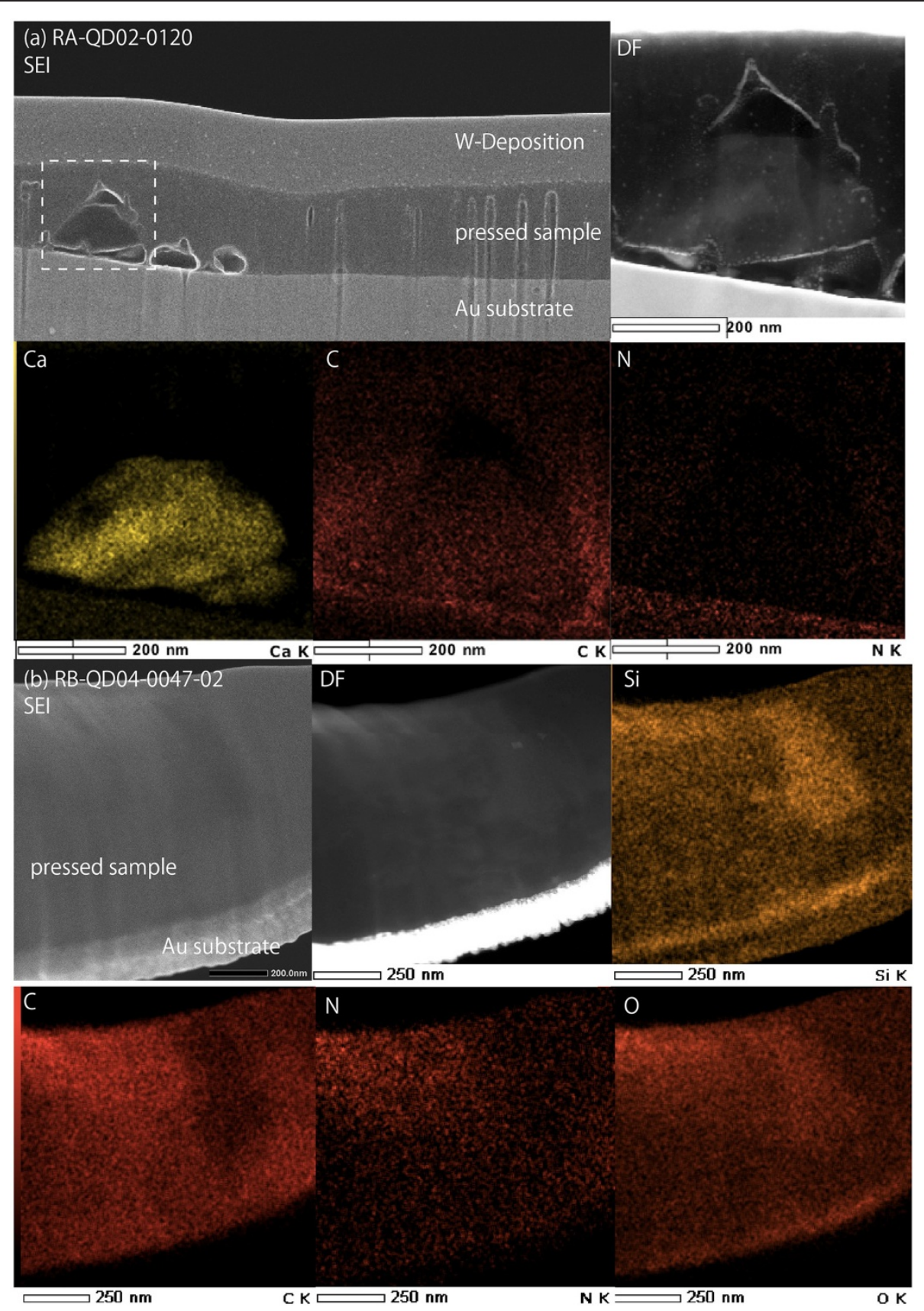

Figure 4 Images obtained from TEM/STEM-EDS (a, b). A dashed box in the secondary electron image (SEl) of RA-QD02-0120 shows the area of dark field (DF) image; and the EDS images show color elemental maps of Si, N, O, C, and Ca. More intense colors correspond to higher concentrations of these elements. Vertical artifacts in the SEl image are from FIB fabrication of the UTS.

category 3 particles to cosmic rays during operation of the spacecraft, which might cause a change in their structure and chemical composition, should also be considered. Collected samples and potential contaminants should also be investigated both before and after irradiation by ultraviolet rays or electron beam for further confirmation of sample origin.

\section{Summary}

The possible origin of the carbonaceous particles in Hayabusa-returned samples was investigated by reconstructing the preliminary examination team for category 3 particles and the flow. Category 3 particles were divided into different types based on their chemical composition and determined that there could have been multiple 


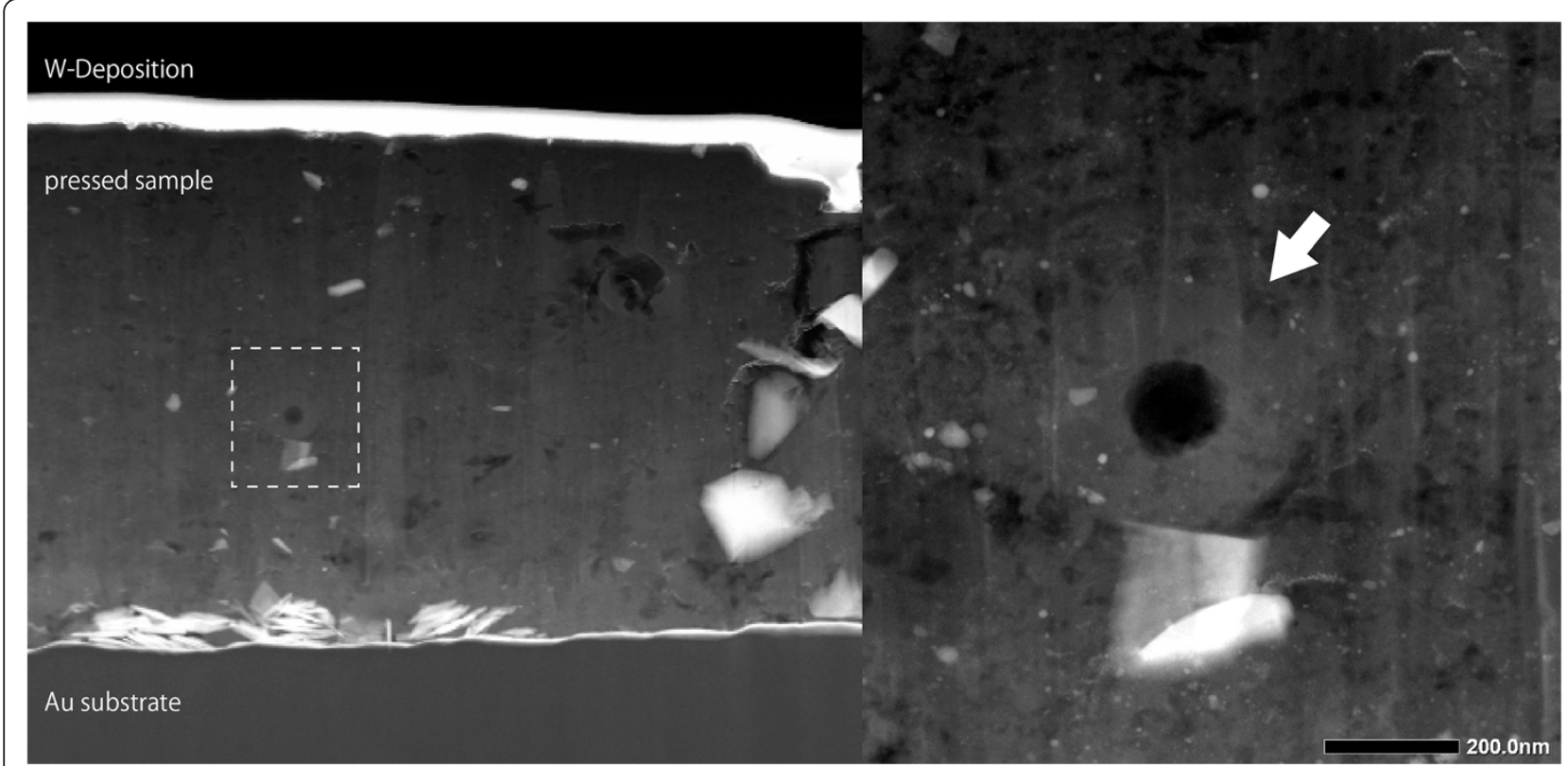

Figure 5 A STEM/dark field image of the UTS of IOM. The right image is a zoomed view of the area in the left image enclosed by the dashed box. The white arrow indicates a nano-globule in the pressed carbon material.

sources of the category 3 particles, although some category 3 particles may have the same origin. In future work, potential contaminants will be investigated using the same analysis flow developed in this study to minimize sample contamination during the optimized second examination.

The new optimized sample-handling system developed through these examinations, including transportation between institutes and the sequence of the analytical flow, will be important not only for the Hayabusa project, but also for sample handling, preparation, and analysis during future sample return missions such as Hayabusa 2 and Osiris-REX.

\section{Abbreviations}

ESCUC: Extraterrestrial Sample Curation Center; BSE: backscattered electron; EDS: energy dispersion spectrometry; FE-SEM: field emission scanning electron microscopy; FIB: focused ion beam; FT-IR: Fourier-transform infrared spectroscopy; HASPET: Hayabusa sample preliminary examination team; IOM: insoluble organic matter; NanoSIMS: nano-secondary ion mass spectrometry; (S)TEM: (scanning) transmission electron microscopy; SFA: spacecraft and fairing assembly building; ToF-SIMS: time-of-flight secondary ion mass spectrometry; UTS: ultra-thin section; XANES: X-ray-absorption near-edge structure.

\section{Competing interests}

The authors declare that they have no competing interests.

\section{Authors' contributions}

MU contributed to sample preparation, TEM analysis, image data interpretation, and manuscript preparation. IO operated TEM analysis. MU, YK, TY, and YI carried out the sample handling, electron beam analyses, and interpretation. MI, HN, FK, HY, YT, HM, YK, TO, and MA participated in the design of the research and interpretation. All authors read and approved the final manuscript.

\section{Acknowledgements}

The authors gratefully acknowledge the reviews by two anonymous reviewers. TEM analyses were performed at JEOL Ltd. XANES analysis was conducted in UVSOR Facility in Institute for Molecular Science, supported by Nanotechnology Platform Program (Molecule and Material Synthesis) of the Ministry of Education, Culture, Sports, Science and Technology (MEXT), Japan.

\section{Author details}

${ }^{1}$ Institute of Space and Astronautical Science (ISAS), Japan Aerospace Exploration Agency (JAXA), 3-1-1 Yoshinodai, Sagamihara, Kanagawa 252-5210, Japan. ${ }^{2}$ Department of Earth and Planetary Science, Faculty of Science, Kyushu University, Hakozaki, Fukuoka 812-8581, Japan. ${ }^{3}$ Kochi Institute for Core Sample Research, Japan Agency for Marine-Earth Science Technology (JAMSTEC), 200 Monobe Otsu, Nankoku, Kochi 783-8502, Japan.

${ }^{4}$ Department of Earth and Space Science Osaka University, 1-1 Machikaneyama, Toyonaka, Osaka 560-0043, Japan. ${ }^{5}$ Department of Biogeochemistry, Japan Agency for Marine-Earth Science and Technology (JAMSTEC), 2-15 Natsushima, Yokosuka 237-0061, Japan. 'Life, Environment and Materials Science, Fukuoka Institute of Technology, 3-30-1 Wajiro-higashi, Fukuoka 811-0295, Japan. 'JEOL Ltd, 1-2, Musashino 3-chome, Akishima, Tokyo 196-8558, Japan. ${ }^{8}$ Natural History Sciences, Hokkaido University, Sapporo 060-0810, Japan.

Received: 30 March 2014 Accepted: 19 August 2014

Published: 26 August 2014

\section{References}

Alexander CM, O'D FM, Yabuta H, Cody GD (2007) The origin and evolution of chondrites recorded in the elemental and isotopic compositions of their macromolecular organic matter. Geochim Cosmochim Acta 71:4380-4403

De Gregorio BT, Stroud RM, Nittler LR, Alexander CM, O'D KALD, Zega TJ (2010) Isotopic anomalies in organic nanoglobules from Comet 81PNWild 2: comparison to Murchison nanoglobules and isotopic anomalies induced in terrestrial organics by electron irradiation. Geochim Cosmochim Acta 74:4454-4470

Ebihara M, Sekimoto S, Shirai N, Hamajima Y, Yamamoto M, Kumagai K, Oura Y, Ireland TR, Kitajima F, Nagao K, Nakamura T, Naraoka H, Noguchi T, Okazaki R, Tsuchiyama A, Uesugi M, Yurimoto H, Zolensky ME, Abe M, Fujimura A, Mukai T, Yada T (2011) Neutron activation analysis of a particle returned from asteroid Itokawa. Science 333:1119-1121 
Garvie LA, Buseck PR (2004) Nanosized carbon-rich grains in carbonaceous chondrite meteorites. Earth Planet Sci Lett 224:431-439

Ito M, Uesugi M, Naraoka H, Yabuta H, Kitajima F, Mita H, Takano Y, Karouji Y, Yada T, Ishibashi Y, Okada T, Abe M (2014) H, C and N isotopic compositions of HAYABUSA Category 3 organic samples. Earth Planet Space 66:91

Kitajima F, Kotsugi M, Ohkochi T, Naraoka H, Ishibashi Y, Abe M, Fujimura A, Okazaki R, Yada T, Nakamura T, Noguchi T, Nagao K, Tsuchiyama A, Mukai T, Sandford SA, Okada T, Shirai K, Ueno M, Yoshikawa M, Kawaguchi J (2011) A micro-spectroscopic approach to the carbonaceous matter in the particles recovered by the HAYABUSA mission, (abstract \#1855). 42nd Lunar and Planetary Science Conference. Houston, 7-11 March 2011, CD-ROM

Kitajima F, Kotsugi M, Ohkochi T, Naraoka H, Ishibashi Y, Uesugi M, Karouji Y, Abe M, Fujimura A, Yada T, Okazaki R, Nakamura T, Noguchi T, Nagao K, Tsuchiyama A, Yurimoto H, Ebihara M, Ito M, Yabuta H, Mita H, Takano Y, Mukai T, Sandford SA, Okada T, Shirai K, Ueno M, Yoshikawa M, Kawaguchi J (2014) A micro-Raman and infrared spectrosopic approach to the several stony and organic (category 3) particles recovered by the Hayabusa mission. Earth Planet Space, in press

Matsumoto T, Tsuchiyama A, Nakamura-Messenger K, Nakano T, Uesugi K, Takeuchi A, Zolensky ME (2013) Three-dimensional observation and morphological analysis of organic nanoglobules in a carbonaceous chondrite using X-ray micro-tomography. Geochim Cosmochim Acta 116:84-95

Nagao K, Okazaki R, Nakamura T, Miura YN, Osawa T, Bajo K, Matsuda S, Ebihara M, Ireland TR, Kitajima F, Naraoka H, Noguchi T, Tsuchiyama A, Yurimoto H, Zolensky ME, Uesugi M, Shirai K, Abe M, Yada T, Ishibashi Y, Fujimura A, Mukai T, Ueno M, Okada T, Yoshikawa M, Kawaguchi J (2011) Irradiation history of Itokawa regolith material deduced from noble gases in the Hayabusa samples. Science 333:1128-1131

Nakamura T, Noguchi T, Tanaka M, Zolensky ME, Kimura M, Tsuchiyama A, Nakato A, Ogami T, Ishida H, Uesugi M, Yada T, Shirai S, Fujimura A, Okazaki R, Sandford SA, Ishibashi Y, Abe M, Okada T, Ueno M, Mukai T, Yoshikawa M, Kawaguchi J (2011) Itokawa dust particles: a direct link between S-type asteroids and ordinary chondrites. Science 333:1113-1116

Nakamura-Messenger K, Zolensky ME, Tomita S, Nakashima S, Tomeoka K (2002) Hollow organic globules in the Tagish Lake meteorite as possible products of primitive organic reactions. Int J Astrobiol 1:179-189

Nakamura-Messenger K, Messenger S, Keller LP, Clemett SJ, Zolensky ME (2006) Organic globules in the Tagish Lake meteorite: remnants of the protosolar disk. Science 314:1439-1442

Naraoka H, Mita H, Hamase K, Mita M, Yabuta H, Saito K, Fukushima K, Kitajima F, Sandford SA, Nakamura T, Noguchi T, Okazaki R, Nagao K, Ebihara M, Yurimoto H, Tsuchiyama A, Abe M, Shirai K, Ueno M, Yada T, Ishibashi Y, Okada T, Fujimura A, Mukai T, Yoshikawa M, Kawaguchi J (2012) Preliminary organic compound analysis of microparticles returned from Asteroid 25143 Itokawa by the Hayabusa mission. Geochem J 46:61-72

Naraoka H, Aoki D, Fukushima K, Uesugi M, Ito M, Kitajima F, Mita H, Yabuta H, Takano Y, Yada T, Ishibashi Y, Okada T, Abe M (2014) ToF-SIMS analysis of carbonaceous particles in the sample capsule of the Hayabusa mission. Earth Planet Space, in press

Noguchi T, Nakamura T, Kimura M, Zolensky ME, Tanaka M, Hashimoto T, Konno M, Nakato A, Ogami T, Fujimura A, Abe M, Yada T, Mukai T, Ueno M, Okada T, Shirai K, Ishibashi Y, Okazaki R (2011) Incipient space weathering observed on the surface of Itokawa dust particles. Science 333:1121-1125

Tsuchiyama A, Uesugi M, Matsushima T, Michikami T, Kadono T, Nakamura T, Uesugi K, Nakano T, Sandford SA, Noguchi R, Matsumoto T, Matsuno J, Nagano T, Imai Y, Takeuchi A, Suzuki Y, Ogami T, Katagiri J, Ebihara M, Ireland TR, Kitajima F, Nagao K, Naraoka H, Noguchi T, Okazaki R, Yurimoto H, Zolensky ME, Mukai T, Abe M, Yada T et al (2011) Three-dimensional structure of Hayabusa samples: origin and evolution of Itokawa regolith. Science 333:1121-1125

Yabuta H, Uesugi M, Naraoka H, Ito M, Kilcoyne ALD, Sandford SE, Ohigashi T, Kitajima F, Mita H, Takano Y, Karouji Y, Yada T, Ishibashi Y, Okada T, Abe M (2014) Molecular compositions of Hayabusa Category 3 carbonaceous particles. Earth Planet Space, in press

Yada T, Fujimura A, Abe M, Nakamura T, Noguchi T, Okazaki R, Nagao K, Ishibashi Y, Shirai K, Zolenskt ME, Sandford S, Okada T, Uesugi M, Karouji Y, Ogawa M, Yakame S, Ueno M, Mukai T, Yoshikawa M, Kawaguchi J (2014) Hayabusa return sample curation in the Planetary Material Sample Curation Facility of JAXA. Meteorite Planet Sci 49:135-153
Yurimoto H, Abe K, Abe M, Ebihara M, Fujimura A, Hashiguchi M, Hashizume K, Ireland TR, Itoh S, Katayama J, Kato C, Kawaguchi J, Kawasaki N, Kitajima F, Kobayashi S, Meike T, Mukai T, Nagao K, Nakamura T, Naraoka H, Noguchi T, Okazaki R, Park C, Sakamoto N, Seto Y, Takei M, Tsuchiyama A, Uesugi M, Wakaki S, Yada T et al (2011) Oxygen isotopic compositions of asteroidal materials returned from Itokawa by the Hayabusa mission. Science 333:1116-1119

doi:10.1186/1880-5981-66-102

Cite this article as: Uesugi et al: Sequential analysis of carbonaceous materials in Hayabusa-returned samples for the determination of their origin. Earth, Planets and Space 2014 66:102.

\section{Submit your manuscript to a SpringerOpen ${ }^{\odot}$ journal and benefit from:}

- Convenient online submission

- Rigorous peer review

- Immediate publication on acceptance

- Open access: articles freely available online

- High visibility within the field

- Retaining the copyright to your article

Submit your next manuscript at $\gg$ springeropen.com 